\title{
Partial reinforcement effects on learning and extinction of place preferences in the water maze
}

\author{
José Prados \\ University of Leicester, Leicester, England \\ AND \\ Joan Sansa and Antonio A. Artigas \\ University of Barcelona, Barcelona, Spain
}

\begin{abstract}
In two experiments, two groups of rats were trained in a navigation task according to either a continuous or a partial schedule of reinforcement. In Experiment 1, animals that were given continuous reinforcement extinguished the spatial response of approaching the goal location more readily than animals given partial reinforcement - a partial reinforcement extinction effect. In Experiment 2, after partially or continuously reinforced training, animals were trained in a new task that made use of the same reinforcer according to a continuous reinforcement schedule. Animals initially given partial reinforcement performed better in the novel task than did rats initially given continuous reinforcement. These results replicate, in the spatial domain, well-known partial reinforcement phenomena typically observed in the context of Pavlovian and instrumental conditioning, suggesting that similar principles govern spatial and associative learning. The results reported support the notion that salience modulation processes play a key role in determining partial reinforcement effects.
\end{abstract}

Neurobiological studies of learning and memory frequently use the Morris swimming pool navigation task (Morris, 1981), in which a rat or mouse has to learn to locate an invisible goal by using some distal landmarks as a reference. The analysis of the mechanisms that control spatial learning in the Morris task is of importance because spatial learning has been argued to be a distinctive form of learning. In their influential book, The Hippocampus As a Cognitive Map, O'Keefe and Nadel (1978) stated that spatial learning involves different, specialized systems from those required for other forms of learning, such as classical and instrumental conditioning. This rather provocative formulation has been providential for the area of spatial learning, and literally hundreds (if not thousands) of experiments have been run to assess it.

Early support for the claimed specificity of spatial learning comes from neurobiological studies that have shown that certain cells preferentially respond to spatial locations (e.g., O'Keefe \& Dostrovsky, 1971). The coordinated activity of place cells results in the establishment of place fields when an animal is exposed to a novel environment. This suggests that spatial information might be processed differently from nonspatial information (see, e.g., Best, White, \& Minai, 2001; Lattal \& Abel, 2000; O'Keefe, 1999). Whether the principles that rule spatial learning are also specific is much more controversial.

Behavioral studies have shown that, at least to a certain extent, spatial learning is determined by principles similar to those involved in other forms of learning. The analysis of the acquisition of the Morris task has shown that spatial learning shares many important properties with Pavlovian conditioning. For example, preexposure to a target stimulus in a Pavlovian preparation retards subsequent conditioning of that stimulus, a result known as latent inhibition. Similarly, preexposure to the array of landmarks that subsequently define the location of a hidden goal in a Morris pool task impairs subsequent learning, an instance of latent inhibition in the spatial domain (see, e.g., Prados, Chamizo, \& Mackintosh, 1999). Furthermore, latent inhibition has been shown to be context specific both in standard conditioning and in the spatial domain (e.g., Prados, Artigas, \& Sansa, 2007). Together with latent inhibition, demonstrations of blocking and overshadowing (e.g., Redhead, Roberts, Good, \& Pearce, 1997; Rodrigo, Chamizo, McLaren, \& Mackintosh, 1997; Sánchez-Moreno, Rodrigo, Chamizo, \& Mackintosh, 1999; Sansa \& Prados, 2003) in the Morris task have strengthened the notion that learning to locate an invisible goal involves processes similar to those involved in the acquisition of standard conditioning tasks. More recently, extinction, or deacquisition, of acquired spatial preferences has also been shown to behave like extinction in standard conditioning preparations (Lattal, Mullen, \& Abel, 2003; Prados, Manteiga, \& Sansa, 2003).

One aspect rarely studied in the spatial domain is the generalization that occurs in classical and instrumental 
conditioning from a particular set of training conditions to a novel set. The conditioned response established to a particular conditioned stimulus (CS), for example, is likely to generalize to other stimuli that have never been paired with the reinforcer, depending on their similarity to the CS, defining a gradient of generalization (e.g., Pavlov, 1927). Some recent studies have reported similar generalization gradients obtained when varying the relative position of two stimuli that defined the location of a goal in a spatial task (Artigas, Aznar-Casanova, \& Chamizo, 2005; Rodrigo, Sansa, Baradad, \& Chamizo, 2006).

Other experiments have shown that a change in the physical properties of the context can also produce a decline in the strength of the response to a CS. In a study by Hall and Honey (1989), for example, rats received food after one CS in one context and after another CS in a different context. When extinction trials took place with the CSs presented in their usual contexts, responding declined more slowly than when the stimuli were presented in the opposite contexts (see also Bonardi, Honey, \& Hall, 1990; Hall \& Honey, 1990; Honey, Willis, \& Hall, 1990). In a series of experiments using the Morris task, Prados et al. (2003) replicated this pattern of results. The rats were trained in the spatial task in two different contexts using two different arrays of cues: A, B, C, and D in Context 1 (the pool surrounded by a set of black curtains) and L, $\mathrm{M}, \mathrm{N}$, and $\mathrm{O}$ in Context 2 (the pool surrounded by a set of striped white and blue curtains). Following training, a group of rats was given extinction trials in the alternative context (i.e., with A, B, C, and D in the striped context and L, M, N, and O in the black context; Group Different), whereas another group of rats was given extinction in the training context (Group Same). During the first extinction trials of the experiment, both Group Same and Group Different showed a marked preference for the platform quadrant (the area where the platform was located during the training), although later on, during the last extinction trials, both groups performed at random level. However, the rats in Group Same spent more time in the platform quadrant than did the rats in Group Different, so an effect of context was observed. This result is what could be expected on the basis of the principle of generalization decrement following a context switch and replicates the context effect that can be observed in standard conditioning tasks.

These demonstrations of context specificity of learning have depended on a change in the physical characteristics of the environment in which learning takes place. But extinction can also be facilitated if it is accompanied by a change in the internal state of the animal. According to a number of theories, the experience of nonreinforcement during extinction generates an internal state of frustration (e.g., Amsel, 1967, 1992) or a memory trace of nonreinforcement (e.g., Capaldi, 1967). When extinction takes place after training with a continuous reinforcement schedule, the introduction of this state or trace for the first time will change the context in which the conditioned response occurs and will thereby facilitate extinction through a generalization decrement. However, training with a partial reinforcement schedule provides an oppor- tunity for the subject to become familiarized with nonreinforcement and thereby reduces generalization decrement. This is the standard explanation for the well-known partial reinforcement extinction effect (PREE). The PREE is one of the most thoroughly studied phenomena in learning and can be defined as a resistance to the detrimental effects of extinction following partial reinforcement. It has been extensively studied in instrumental learning (see Mackintosh, 1974, for a review) and has also been demonstrated within Pavlovian conditioning (e.g., Haselgrove, Aydin, \& Pearce, 2004; Pearce, Redhead, \& Aydin, 1997; Rescorla, 1999).

The experiments reported here assessed whether the well-known effects of partial reinforcement in standard conditioning tasks could be proven to occur in the spatial domain. In Experiment 1, we assessed the effect of partial reinforcement on extinction of an acquired spatial preference. In Experiment 2, we investigated the effect of partial reinforcement on acquisition of a second spatial task using the same reinforcer. In these experiments, we aimed not only to improve our knowledge of partial reinforcement effects on learning and extinction but also to increase our understanding about the relationship between spatial and other forms of learning, such as Pavlovian and instrumental conditioning.

\section{EXPERIMENT 1}

Experiment 1 was designed to verify whether the PREE could be shown to occur in the spatial domain. If instrumental, Pavlovian, and spatial learning are governed by similar principles, partial reinforcement can be expected to retard extinction of a learned spatial preference in a navigation task. To evaluate this hypothesis, two groups of rats were trained in the Morris pool according to a partial or continuous reinforcement schedule (Groups Partial and Continuous, respectively). After completion of the escape training phase, all of the animals were given 10 extinction trials in which the invisible platform was removed from the pool - a procedure that proved to be suitable for the study of extinction in the spatial domain (Prados et al., 2003).

\section{Method}

Subjects. The subjects were 16 Long-Evans rats (Rattus norvegicus), 8 males and 8 females. They had previously participated in an experiment that involved taste aversion conditioning and were approximately 6 months old at the start of the experiment. The rats were housed in pairs in a room that was lit for $12 \mathrm{~h}$ each day. They were tested at the same time on successive days during the period when their living quarters were lit. Rats were assigned at random (4 males and 4 females each) to two groups: Continuous and Partial.

Apparatus. The apparatus was a large circular swimming pool, made of plastic and fiberglass, modeled after that used by Morris (1981). It measured $1.58 \mathrm{~m}$ in diameter and $0.65 \mathrm{~m}$ deep, and it was filled to a depth of $49 \mathrm{~cm}$ with water that was made opaque by adding $750 \mathrm{ml}$ of polystyrene latex, a nontoxic substance that does not spoil. A constant temperature of $21^{\circ} \mathrm{C} \pm 1^{\circ}$ was maintained. The pool was situated in the middle of a large room, mounted on a wooden base $0.43 \mathrm{~m}$ above the floor. A circular platform, mounted on a rod and base, $0.11 \mathrm{~m}$ in diameter and made of transparent Perspex, could be placed in one quadrant of the pool, $0.38 \mathrm{~m}$ from the side, with its top $1 \mathrm{~cm}$ below the surface of the water. It was thus invisible for the animals. The pool was surrounded by a set of black curtains reaching 
from ceiling to $0.50 \mathrm{~m}$ above the floor; the curtains formed a circular enclosure $2.40 \mathrm{~m}$ in diameter. There was a lighting system attached to a false ceiling and made up of six low-consumption 70 -W lightbulbs, $1.75 \mathrm{~m}$ above the pool. A closed-circuit video camera with a wide-angle lens was mounted in the center of the false ceiling, and its picture was relayed to recording equipment in an adjacent room.

We used a set of four landmarks, placed at equal distances around the circumference of the pool. The landmarks were (A) a 40-W light placed inside a white plastic inverted cone $11 \mathrm{~cm}$ in height and $13 \mathrm{~cm}$ in diameter at the base, (B) a plastic beach ball $30 \mathrm{~cm}$ in diameter with alternate blue-white, yellow-white, and orange-white vertical segments, (C) an intermittent 1-W light flashing on and off at a frequency of 60-80 times per minute, and (D) a green plastic plant, approximately $35 \mathrm{~cm}$ in diameter and $30 \mathrm{~cm}$ in height. The landmarks were suspended from the ceiling, $35 \mathrm{~cm}$ above the surface of the water and with their midline directly above the wall of the pool. The location of the platform was always between $\mathrm{C}$ and $\mathrm{D}$.

Procedure. There were two phases in Experiment 1: escape training and extinction. During the escape training phase, all of the animals were trained to swim toward an invisible platform located in a fixed position relative to the four landmarks (A, B, C, and D). Animals in Group Continuous were given 10 days of escape training at a rate of four escape trials per day with an average intertrial interval (ITI) of $15 \mathrm{~min}$. Escape training trials consisted of placing the rat into the pool with the four landmarks (A, B, C, and D) and the invisible platform present. Each rat was given $60 \mathrm{sec}$ to find the platform, where it was allowed to stay for $30 \mathrm{sec}$. If a rat failed to find the platform within $60 \mathrm{sec}$, the experimenter placed his or her thumb about $5 \mathrm{~cm}$ in front of the rat and guided it to the platform, where it was allowed to stay for $30 \mathrm{sec}$. During each session, the rat was placed in the pool once in each of the four possible start points, each one below one of the four landmarks-A, B, C, and D-that defined the platform location. The order of the start points was determined at random within each session, in such a way that there was a long and a short path to the platform (A and C, for example) in every block of two trials throughout the experiment (i.e., AD BC, CA DB, DB CA, BC AD, etc.). The entire array of landmarks and the platform location were rotated $90^{\circ}$ counterclockwise on every trial throughout the experiment. The time spent by the rats in finding the platform (latency of escape) was recorded.

Animals in Group Partial were also given 10 days of escape training at a rate of four escape trials per day. However, in addition to the four escape trials, these animals were also given two nonreinforced trials. During the nonreinforced trials, the rats were allowed to swim for $60 \mathrm{sec}$ all around the pool, while we registered the time spent in each of four previously defined quadrants: the platform training (PT) quadrant, delimited by Landmarks C and D, and the other three. The order of reinforced and nonreinforced trials was varied in every session in such a way that the nonreinforced trials were presented three times in the first, third, fourth, and sixth positions, and four times in the second and fifth positions.

On the day after the last day of escape training, all of the animals were given 2 escape retraining trials, which were followed immediately by 10 extinction trials with an average ITI of $15 \mathrm{~min}$. The procedure for extinction trials was exactly the same as for nonreinforced trials in the previous phase of the experiment.

\section{Results and Discussion}

The results of the escape training phase are shown in Figure 1, with those for reinforced trials in the left-hand panel and those for the nonreinforced trials (Group Partial) in the right-hand panel. During the reinforced trials, the two groups showed a similar pattern of results. An ANOVA conducted on the data with group and blocks of two sessions as factors showed a significant effect of blocks $[F(4,56)=18.19]$, whereas there were no differences between groups and no significant group $\times$ blocks

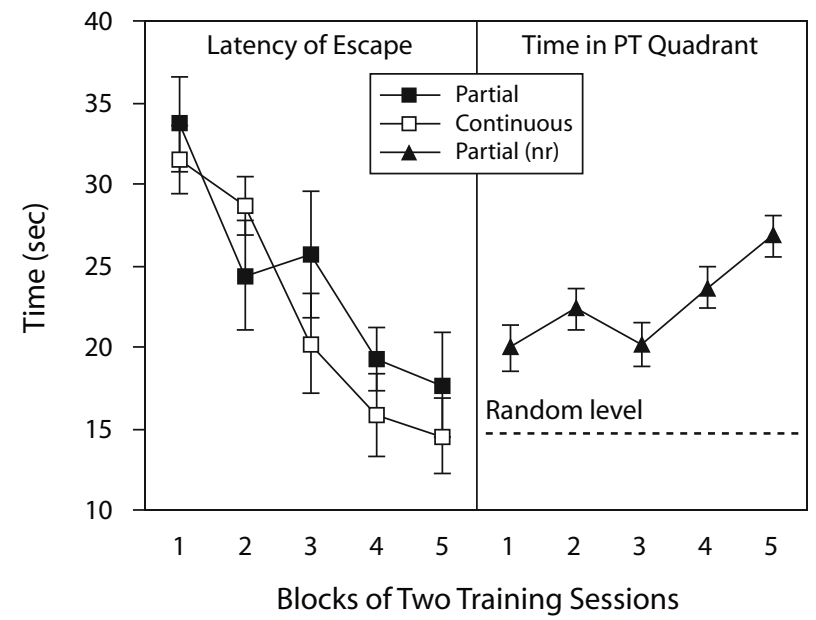

Figure 1. Mean latency $( \pm S E)$ to find the platform over blocks of two sessions during the escape training phase of Experiment 1 (left panel); mean time $( \pm S E)$ spent in the platform training (PT) quadrant during the nonreinforced (nr) trials in Group Partial (right panel).

interaction [maximum $F(4,56)=1.38]$. (Here and elsewhere, a significance level of $p<.05$ was adopted.) On the other hand, one-sample $t$ tests carried out on the data corresponding to the nonreinforced trials (Group Partial) showed that animals performed above chance in every block of two sessions [minimum $t(7)=3.53$ ].

Group latency of escape means during the two retraining trials that preceded extinction were $15.31 \mathrm{sec}(S E M=$ $2.37)$ and $14.06 \mathrm{sec}(S E M=2.71)$ for Group Continuous and Group Partial, respectively. A one-way ANOVA conducted on these data showed that there were no differences between groups $(F<1)$. The results of the extinction phase are shown in Figure 2. The animals in Group Continuous extinguished more readily than the animals of Group Partial. These impressions were confirmed by

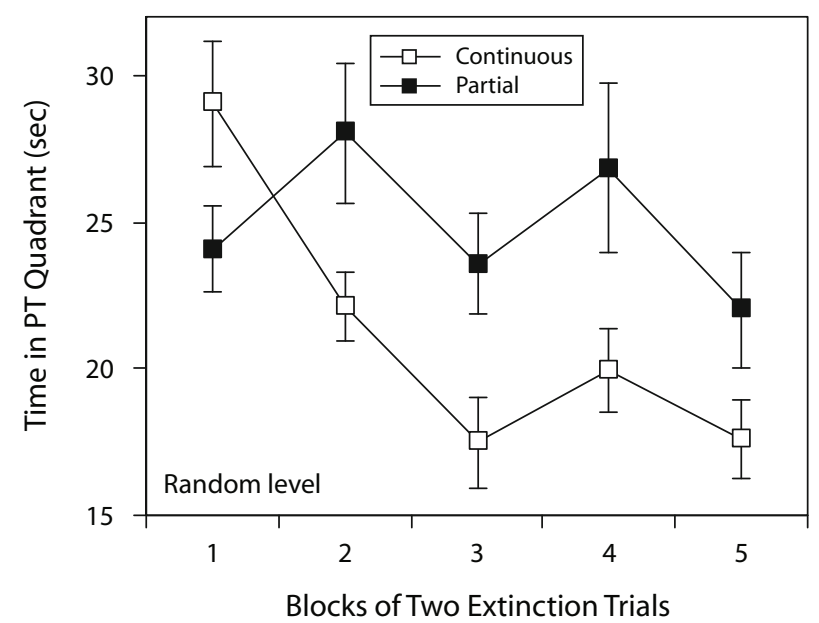

Figure 2. Mean time in the platform training (PT) quadrant $( \pm S E)$ over blocks of two trials during the extinction phase of Experiment 1. 
statistical analyses. An ANOVA conducted on the data with group and blocks of two extinction trials as factors showed significant effects of group $[F(1,14)=4.64]$ and of blocks $[F(4,56)=6.52]$ and a significant group $\times$ blocks interaction $[F(4,56)=4.72]$. Further analyses of this interaction (simple main effects) showed that groups significantly differed in Blocks 2,3 , and $4[F \mathrm{~s}(1,14)>$ 4.54]. Also, the blocks factor was significant in both groups $[F \mathrm{~s}(4,56)>2.83]$.

The present results add to those of previous studies that have demonstrated similarities between spatial learning and other forms of learning, such as instrumental and Pavlovian conditioning. The detrimental effect of partial reinforcement on extinction of an acquired spatial preference mimics the well-documented effect of partial reinforcement on extinction of instrumental and conditioned responses.

Partial reinforcement effects have been attributed traditionally to generalization decrement (e.g., Amsel, 1967, 1992; Capaldi, 1967). According to this view, what is learned during partial reinforcement is better able to generalize to the extinction phase in which reinforcement is withdrawn. In other words, experience of nonreinforcement during the acquisition phase of the experiment makes it hard for the animals in the partial reinforcement condition to detect the difference between acquisition and extinction. On the other hand, the novelty of nonreinforcement in the group given continuous reinforcement facilitates generalization decrement at the time of extinction. The decrement in performance can then be attributed, following generalization decrement theories, to the change from the conditions of acquisition.

Although generalization decrement offers a good explanation for the PREE, it is not clear whether it can account for other partial reinforcement effects that have been reported in Pavlovian conditioning. According to generalization decrement theories, the key factor that accounts for the PREE is the similarity between the acquisition and extinction phases of the experiments. By the same token, animals trained according to a continuous reinforcement schedule should show more generalization when trained in a novel task using continuous reinforcement than would animals previously trained according to a partial reinforcement schedule. In a series of experiments using a conditioned emotional response procedure, Hall, Prados, and Sansa (2005) found a pattern of results that seems to challenge this prediction. In their experiments, a group of rats was given conditioning trials in which a given cue, A, was consistently paired with a shock unconditioned stimulus (US; $\mathrm{A}+$, a continuous reinforcement procedure, where " $\mathrm{A}$ " represents the signal, either a light or a tone, and "+" represents the shock). A second group of rats received the same pairings of the signal A and the shock, alternated with presentations of $\mathrm{A}$ alone (A+/A training, a partial reinforcement procedure). This was followed by a test stage in which a new signal, B, was paired with the shock according to a continuous reinforcement schedule. The results showed that the animals given partial reinforcement with Cue A learned the relationship between B and the shock US faster than animals given continuous reinforcement with Cue A. As suggested above, this result is the opposite of what could be expected from a generalization decrement view of partial reinforcement effects. Hall et al. (2005) suggested an alternative explanation based on salience modulation processes.

Traditionally, it has been supposed that salience is determined by physical intensity and is a fixed stimulus property (e.g., Rescorla \& Wagner, 1972). Salience is, however, a much more interesting psychological concept that reflects the effectiveness of the stimulus - how it affects an organism. Furthermore, recent research has shown that the effective salience of a stimulus can be modified by experience (e.g., Blair \& Hall, 2003; Hall, 2003). Hall suggested that mere exposure to a stimulus could be expected to reduce its effective salience - a process of habituation. He also suggested that this process could be reversed by presentations of a cue associated with the stimulus. That is, associative activation of a stimulus (in the absence of the stimulus itself) will act to restore its salience. Hall et al. (2005) argued that, in the continuous reinforcement condition of their experiments, presentations of the shock consistently signaled by Cue A would result in a loss of effective salience by the shock US. On the contrary, in the partial reinforcement condition, presentations of Cue A alone would result in associative activation of the shock representation and would, thus, alleviate the loss of effective salience produced by shock presentations in A+ trials. According to the authors of the study, better performance in the test with a novel Cue B paired with the shock reflects an effective salience of the shock after partial reinforcement higher than after continuous reinforcement.

The hypothesis put forward by Hall (2003) suggests that differential changes in the effective salience of stimuli that occur during continuous and partial reinforcement could also play a role in determining the effects of continuous and partial reinforcement on extinction of learned behavior. According to that view, continuous reinforcement decreases the effectiveness of the reinforcer, whereas partial reinforcement maintains its salience or effectiveness at a high level. Assuming that the conditioned response relies on the salience or effectiveness of the reinforcer, animals in the partial reinforcement condition could be expected to show a more persistent pattern of response than would animals in the control condition. Experiment 2 was designed to assess the merits of this hypothesis by examining whether, in the spatial domain, associative activation of the reinforcer maintains its salience or effectiveness at a high level.

\section{EXPERIMENT 2}

In Experiment 2, we replicated the design employed by Hall et al. (2005) described above but used the Morris swimming pool navigation task instead of a standard Pavlovian preparation. Two groups of rats were trained to locate an invisible goal, whose position was defined by several landmarks. In the first phase of the experiment, the platform was signaled by the presence of a salient 
stimulus, Z, located immediately above the hidden platform; in that way, the platform could be reached simply by moving toward the stimulus - a guide task (O'Keefe \& Nadel, 1978), also referred to as a beacon homing strategy (Gallistel, 1990; Redhead et al., 1997). Animals in Group Continuous were trained in the guide task according to a continuous reinforcement schedule, whereas animals in Group Partial were given partial reinforcement training. After completion of the first escape training phase, all of the animals were trained in a new task that involved learning to locate the hidden platform in the presence of a new set of landmarks-A, B, C, and D-that were some distance from the goal over the edge of the platform-a cartographic strategy (O'Keefe \& Nadel, 1978), also referred to as a piloting strategy (e.g., Gallistel, 1990; Redhead et al., 1997). Training with the landmarks took place according to a schedule of continuous reinforcement. According to the hypothesis under examination, associative activation of the omitted reinforcer during the nonreinforced trials of the partial schedule could be expected to maintain its salience or effectiveness at a high level. Consequently, learning of the second task in the presence of the landmarks should proceed more readily in Group Partial than in Group Continuous. Alternatively, according to a generalization decrement view, higher similarity between the two phases of the experiment would allow better performance in Group Continuous than in Group Partial.

\section{Method}

Subjects and Apparatus. A total of 16 male hooded Long-Evans rats were used and maintained as in the previous experiment. They were divided into two groups $(n=8)$ : Continuous and Partial. The apparatus was the same as that in Experiment 1. A new, proximal landmark was used, $\mathrm{Z}$ : a white sphere, $10 \mathrm{~cm}$ in diameter, suspended from the ceiling, $30 \mathrm{~cm}$ immediately above the escape platform.

Procedure. There were two phases in Experiment 2: escape training with landmark $Z$, and escape training with landmarks A, B, C, and $\mathrm{D}$. During the escape training with $\mathrm{Z}$, all of the rats were given 12 days of escape training at a rate of four escape trials per day. In addition to these four escape trials, animals in Group Partial were also given two nonreinforced trials. The order of reinforced and nonreinforced trials was varied in every session in such a way that the nonreinforced trials were presented four times in every position. The location of the platform (and the $\mathrm{Z}$ cue) varied from trial to trial among four possible positions within the pool (corresponding to the center of four quadrants, NW, NE, SE, and SW).

During the second phase of the experiment, all of the animals were given escape training with Landmarks A, B, C, and D according to a continuous reinforcement schedule. All of the animals were given four sessions of training at a rate of four escape trials per session. The location of the platform was always between $\mathrm{C}$ and $\mathrm{D}$.

\section{Results and Discussion}

The results of the escape training with $\mathrm{Z}$ are shown in Figure 3, with those for reinforced trials in the left-hand panel and those of the nonreinforced trials (Group Partial) in the right-hand panel. During the reinforced trials, the two groups showed similar patterns of results. An ANOVA conducted on the data with group and blocks of two sessions as factors showed a significant effect of blocks $[F(5,70)=$ 34.04], but there were no differences between groups and

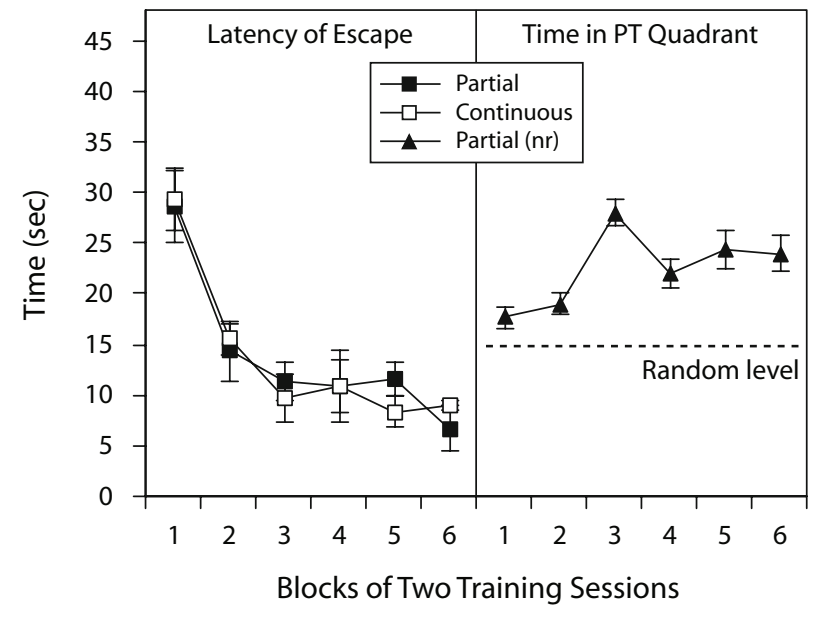

Figure 3. Mean latency $( \pm S E)$ to find the platform over blocks of two sessions during the escape training phase with $\mathrm{Z}$ of Experiment 2 (left panel); mean time $( \pm S E)$ spent in the platform training (PT) quadrant during the nonreinforced (nr) trials in Group Partial (right panel).

no significant group $\times$ blocks interaction $(F \mathrm{~s}<1)$. On the other hand, one-sample $t$ tests carried out on the data corresponding to the nonreinforced trials (Group Partial) showed that animals performed above chance in every block of two sessions [minimum $t(7)=2.42$ ].

The results of the escape training with Landmarks A, B, $\mathrm{C}$, and $\mathrm{D}$ are shown in Figure 4. Rats previously trained to find the platform by swimming toward the $\mathrm{Z}$ cue according to a partial reinforcement schedule learned faster than animals given the same training according to a continuous reinforcement schedule. An ANOVA conducted on the data with group, sessions, and blocks of two escape trials as factors showed significant effects of group $[F(1,14)=$ 5.07] and blocks $[F(1,14)=7.14]$. The remaining factors and interactions were all nonsignificant [maximum $F(3,42)=2.82]$.

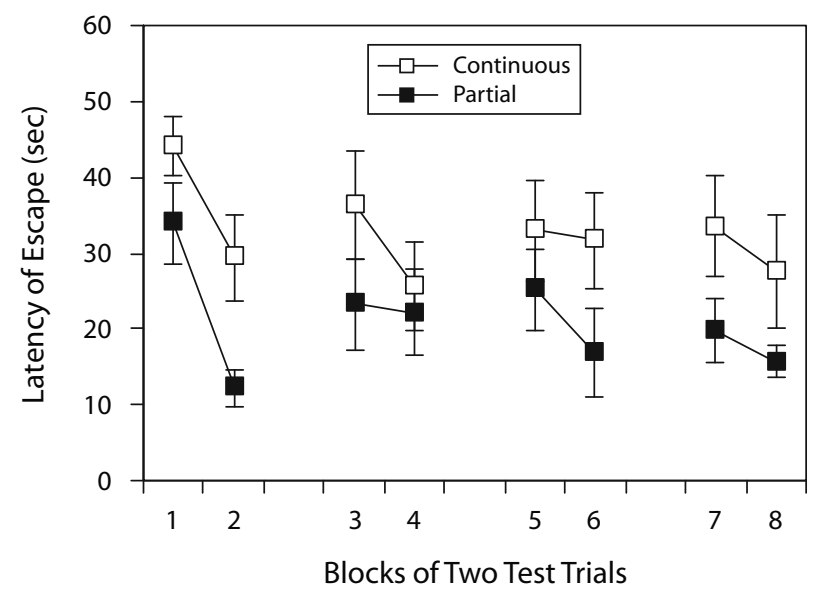

Figure 4. Mean latency $( \pm S E)$ to find the platform over blocks of two sessions during the escape training phase with Landmarks A, B, C, and D of Experiment 2. 
The present results show that training in a spatial task according to a partial reinforcement schedule benefits subsequent learning of a different spatial task that makes use of the same reinforcer. The pattern of results observed in the present experiment replicates previous findings obtained in a Pavlovian preparation (Hall et al., 2005).

The finding that partial reinforcement benefits subsequent learning according to a continuous reinforcement schedule might be taken to challenge traditional theories of partial reinforcement based on generalization decrement: Greater similarity between the two phases of the experiment should benefit learning in Group Continuous compared with Group Partial. The pattern of results observed corresponds, however, to what can be predicted from an alternative view that takes into account salience modulation processes. Regular omission of the reinforcer during the first phase of the experiment could have contributed to maintaining its salience or effectiveness at a high level. That being the case, the reinforcer would have a greater impact in Group Partial than in Group Continuous at the outset of the second phase of the experiment. The fact that rats in Group Partial performed better than animals in Group Continuous since the start of the test phase with Landmarks A, B, C, and D seems to support this view.

\section{GENERAL DISCUSSION}

In two experiments, rats were trained in a navigation task according to either a continuous or a partial schedule of reinforcement. In Experiment 1, after learning to swim toward a platform whose location was defined by four landmarks, rats were given extinction trials in which the platform was removed from the pool. All of the animals showed a marked preference for the platform quadrant during the first extinction trials. Although, later on, rats' performances gradually decreased to chance, the animals given training according to a partial reinforcement schedule showed a greater resistance to the detrimental effects of extinction than did the animals given continuous reinforcement. This result can be taken to be a demonstration of the well-known PREE in the Morris pool task. As far as we know, this is a novel finding within the spatial domain that adds to previous findings (Lattal et al., 2003; Prados et al., 2003), suggesting that extinction of spatial preferences mimics extinction in standard conditioning preparations.

In Experiment 2, we examined the effect of partial reinforcement on acquisition of a second task using the same reinforcer. Animals were trained to locate the invisible platform by swimming toward a salient stimulus placed immediately above the platform. Following this training, they were then required to learn the location of the platform by using a set of novel, distal landmarks. Those rats that were given initial training according to a partial reinforcement schedule showed better performance in the new task than did those animals initially trained according to a continuous reinforcement schedule. This finding replicates the pattern of results reported by Hall et al. (2005), who used a standard Pavlovian preparation, suggesting that similar principles rule spatial learning and Pavlovian conditioning.

As argued above, the PREE observed in Experiment 1 could be easily explained in terms of generalization decrement: Familiarity with nonreinforcement (and its effects, such as frustration) in the partial reinforcement condition can be expected to diminish the change of context that can be expected to occur in the transition from acquisition to extinction. On the other hand, the results observed in Experiment 2, in which the animals were required to learn a novel task using the same reinforcer, do not seem consistent with predictions of these theories. Greater similarity between the two phases of the experiment would lead us to expect less generalization decrement in the group that was always given continuous reinforcement, resulting in better learning of the second task in Group Continuous than in Group Partial. This, however, was not the result found in the present Experiment 2-nor in the original experiments reported by Hall et al. (2005) using a Pavlovian preparation.

The state of frustration has been argued to have other effects that might be relevant to account for partial reinforcement phenomena. According to Amsel (1958; see also Ross, 1964), in the absence of reinforcement, a state of frustration would elicit a withdrawal response that would compete with any instrumental response of approaching the cues signaling the reward. In a group of animals given continuous reinforcement, the absence of reward at the time of extinction would then elicit an interfering response that would decrease the instrumental response - thus favoring extinction. In contrast, animals given partial reinforcement can be expected to experience frustration during the acquisition phase of the experiment. That being the case, given that animals will experience frustration in some trials in the presence of the reward, counterconditioning might develop, by which frustration would come to elicit the instrumental response of approaching the cues that inform about the reward. In the present Experiment 1, if frustration had become an elicitor of the response of approaching the location of the hidden platform, the experience of this emotional state during the extinction phase of the experiment would have encouraged animals in Group Partial to keep searching, thus retarding extinction. The results of Experiment 2 are a bit more puzzling: The animals were tested in the absence of the frustration state - according to a continuous reinforcement schedule - and no differences should be expected between the two groups. It could be argued, however, that given that the rats did not immediately find the hidden platform, at least during the first trials of the test phase, a certain amount of frustration would be present during the final critical phase of the experiment. In this case, frustration in Group Partial would elicit the previously learned response. It is not clear whether that would benefit performance in the second task: In the first phase of Experiment 2 , animals were trained in a guide task in which they had to approach a visible cue, whereas in the final test, they had to infer the position of the platform by using distal cues - a piloting strategy. If anything, a rat's approach 
to any of the available cues in the final test would interfere with its finding the hidden platform. Although very attractive, the counterconditioning of frustration theory seems to be not entirely adequate to account for the whole pattern of results reported here.

An alternative explanation for partial reinforcement effects has been proposed by Rescorla $(1999,2001)$. Instead of generalization decrement or counterconditioning, Rescorla focuses on the associative change that occurs during extinction. After training under a continuous reinforcement schedule, the surprising experience of nonreinforcement has been found to decrease the associative strength of the CS. Partial reinforcement, however, by familiarizing the animals with nonreinforcement, reduces the surprise (i.e., the effectiveness) of the nonreinforcement event, attenuating the decrease in associative strength that results from extinction trials (Rescorla, 1999). This interpretation has no difficulties dealing with the PREE found in the present Experiment 1. However, it is not clear whether it can also be applied to Experiment 2. Although animals in Group Continuous and Group Partial had the same experience with the reinforcer, it might be possible that the omission of nonreinforcement during the training in the second task would increase the surprise or effectiveness of the reinforcer. This could be expected to improve the performance of Group Partial in locating the invisible platform during the second phase of the experiment. The problem is that, in the absence of additional supporting evidence, this interpretation could be said to be speculative.

A similar but more convincing way to interpret the present results in terms of effectiveness of the reinforcer takes into account salience modulation processes (Hall, 2003). The advantage of this interpretation is that it provides a detailed account of the circumstances that lead to differential changes in the effectiveness of the reinforcer in the continuous and the partial conditions (see Hall et al., 2005 , for a detailed discussion). According to this view, direct activation of the node representing a particular event can be expected to diminish its sensitivity, reducing the salience or perceptual effectiveness of the event. However, associative activation of the event has been shown to restore the lost effectiveness (e.g., Blair \& Hall, 2003). Thus, associative activation of the reinforcer in the partial reinforcement schedule should maintain its effectiveness at a high level. In the present Experiment 1, greater salience or effectiveness of the reinforcer (escaping from water to a platform) after partial reinforcement would have promoted a persistent pattern of response, compared with the one shown by the animals in the control condition. Similarly, the presence of a more effective reinforcer in the second task of Experiment 2 would have resulted in stronger and more rapid learning after partial reinforcement than it would after continuous reinforcement.

To conclude, the present results show that learning and extinction in the Morris pool navigation task are consistent with predictions from learning theories that have been developed to account for Pavlovian and instrumental conditioning, strengthening the notion that similar processes underlie spatial and nonspatial associative learning.

\section{AUTHOR NOTE}

This work was supported by a grant from the Spanish Ministerio de Educación y Ciencia to the authors. Correspondence concerning this article should be addressed to J. Prados, School of Psychology, University of Leicester, Lancaster Road, Leicester LE1 9HN, England (e-mail: jpg19@1e.ac.uk).

\section{REFERENCES}

Amsel, A. (1958). The role of frustrative nonreward in noncontinuous reward situations. Psychological Bulletin, 55, 102-119.

Amsel, A. (1967). Partial reinforcement effects in vigor and persistence: Advances in frustration theory derived from a variety of within-subjects experiments. In K. W. Spence \& J. T. Spence (Eds.), The psychology of learning and motivation (Vol. 1, pp. 1-65). New York: Academic Press.

Amsel, A. (1992). Frustration theory: An analysis of dispositional learning and memory. Cambridge: Cambridge University Press.

Artigas, A. A., Aznar-Casanova, J. A., \& Chamizo, V. D. (2005). Effects of absolute proximity between landmark and platform in a virtual Morris pool task with humans. International Journal of Comparative Psychology, 18, 224-239.

Best, P. J., White, A. M., \& Minai, A. (2001). Spatial processing in the brain: The activity of hippocampal place cells. Annual Review of Neuroscience, 24, 459-486.

Blair, C. A. J., \& Hall, G. (2003). Perceptual learning in flavor aversion: Evidence for learned changes in stimulus effectiveness. Journal of Experimental Psychology: Animal Behavior Processes, 29, 39-48.

Bonardi, C., Honey, R. C., \& Hall, G. (1990). Context specificity of conditioning in flavor-aversion learning: Extinction and blocking tests. Animal Learning \& Behavior, 18, 229-237.

CAPALDI, E. J. (1967). A sequential theory of instrumental training. In K. W. Spence \& J. T. Spence (Eds.), The psychology of learning and motivation (Vol. 1, pp. 67-156). New York: Academic Press.

Gallistel, C. R. (1990). The organization of learning. Cambridge, MA: MIT Press.

HaLl, G. (2003). Learned changes in the sensitivity of stimulus representations: Associative and nonassociative mechanisms. Quarterly Journal of Experimental Psychology, 56B, 43-55.

Hall, G., \& Honey, R. C. (1989). Contextual effects in conditioning, latent inhibition, and habituation: Associative and retrieval functions of contextual cues. Journal of Experimental Psychology: Animal Behavior Processes, 15, 232-241.

Hall, G., \& Honey, R. C. (1990). Context-specific conditioning in the conditioned-emotional-response procedure. Journal of Experimental Psychology: Animal Behavior Processes, 16, 271-278.

Hall, G., Prados, J., \& Sansa, J. (2005). Modulation of the effective salience of a stimulus by direct and associative activation of its representation. Journal of Experimental Psychology: Animal Behavior Processes, 31, 267-276.

Haselgrove, M., Aydin, A., \& Pearce, J. M. (2004). A partial reinforcement extinction effect despite equal rates of reinforcement during Pavlovian conditioning. Journal of Experimental Psychology: Animal Behavior Processes, 30, 240-250.

Honey, R. C., Willis, A., \& Hall, G. (1990). Context specificity in pigeon autoshaping. Learning \& Motivation, 21, 125-136.

LATTAL, K. M., \& ABEL, T. (2000). Cellular and molecular mechanisms of learning and memory. In M. E. A. Reith (Ed.), Cerebral signal transduction: From first to fourth messengers (pp. 27-71). Totaway, NJ: Humana.

LatTAl, K. M., Mullen, M. T., \& Abel, T. (2003). Extinction, renewal, and spontaneous recovery of a spatial preference in the water maze. Behavioral Neuroscience, 117, 1017-1028.

Mackintosh, N. J. (1974). The psychology of animal learning. London: Academic Press.

Morris, R. G. M. (1981). Spatial localization does not require the presence of local cues. Learning \& Motivation, 12, 239-260.

O'KeEFE, J. (1999). Do hippocampal pyramidal cells signal non-spatial as well as spatial information? Hippocampus, 9, 352-364.

O'KeEfe, J., \& Dostrovsky, J. (1971). The hippocampus as a spatial map: Preliminary evidence from unit activity in the freely-moving rat. Brain Research, 34, 171-175. 
O'KeEFe, J., \& NADEL, L. (1978). The hippocampus as a cognitive map. Oxford: Oxford University Press, Clarendon Press.

Pavlov, I. P. (1927). Conditioned reflexes: An investigation of the physiological activity of the cerebral cortex (G. V. Anrep, Trans.). London: Oxford University Press.

Pearce, J. M., Redhead, E. S., \& Aydin, A. (1997). Partial reinforcement in appetitive Pavlovian conditioning with rats. Quarterly Journal of Experimental Psychology, 50B, 273-294.

Prados, J., Artigas, A. A., \& Sansa, J. (2007). Preexposure effects in the spatial domain: Dissociation between latent inhibition and perceptual learning. Journal of Experimental Psychology: Animal Behavior Processes, 33, 115-123.

Prados, J., Chamizo, V. D., \& Mackintosh, N. J. (1999). Latent inhibition and perceptual learning in a swimming-pool navigation task. Journal of Experimental Psychology: Animal Behavior Processes, 25, 37-44.

Prados, J., Manteiga, R. D., \& Sansa, J. (2003). Recovery effects after extinction in the Morris swimming pool navigation task. Learning \& Behavior, 31, 299-304.

Redhead, E. S., Roberts, A., Good, M., \& Pearce, J. M. (1997). Interaction between piloting and beacon homing by rats in a swimming pool. Journal of Experimental Psychology: Animal Behavior Processes, 23, 340-350.

Rescorla, R. A. (1999). Partial reinforcement reduces the associative change produced by nonreinforcement. Journal of Experimental Psychology: Animal Behavior Processes, 25, 403-414.
Rescorla, R. A. (2001). Experimental extinction. In R. R. Mowrer \& S. B. Klein (Eds.), Handbook of contemporary learning theories (pp. 119-154). Mahwah, NJ: Erlbaum.

Rescorla, R. A., \& Wagner, A. R. (1972). A theory of Pavlovian conditioning: Variations in the effectiveness of reinforcement and non-reinforcement. In A. H. Black \& W. F. Prokasy (Eds.), Classical conditioning II: Current research and theory (pp. 64-99). New York: Appleton-Century-Crofts.

Rodrigo, T., Chamizo, V. D., McLaren, I. P. L., \& Mackintosh, N. J. (1997). Blocking in the spatial domain. Journal of Experimental Psychology: Animal Behavior Processes, 23, 110-118.

Rodrigo, T., SANSA, J., Baradad, P., \& Chamizo, V. D. (2006). Generalization gradients in a navigation task with rats. Learning \& Motivation, 37, 247-268.

Ross, R. R. (1964). Positive and negative partial-reinforcement extinction effects carried through continuous reinforcement, changed motivation, and changed response. Journal of Experimental Psychology, $\mathbf{6 8}, 492-502$.

Sánchez-Moreno, J., Rodrigo, T., Chamizo, V. D., \& Mackintosh, N. J. (1999). Overshadowing in the spatial domain. Animal Learning \& Behavior, 27, 391-398.

Sansa, J., \& Prados, J. (2003). Overshadowing between landmarks in a navigation task. Psicológica, 24, 17-29.

(Manuscript received August 6, 2007; revision accepted for publication March 16, 2008.) 